\title{
VIERA Y CLAVIJO \\ EN LA MIRADA DE AGUSTÍN ESPINOSA
}

\author{
Victoria Galván González \\ Casa-Museo Pérez Galdós, Las Palmas de Gran Canaria
}

\section{RESUMEN}

La dimensión crítica de Agustín Espinosa hacia la tradición literaria canaria cobra gran relieve en su conferencia sobre Viera y Clavijo, "Sobre el signo de Viera», pronunciada con motivo del segundo centenario de su nacimiento, en 1932, en el Círculo de Bellas Artes de Tenerife. Se analiza en estas páginas la mirada de Espinosa del ilustrado arcediano.

Palabras Clave: tradición literaria insular, siglo Xvini, crítica literaria de Agustín Espinosa.

\author{
VIERA Y CLAVIJO \\ IN AGUSTÍN ESPINOSA'S GAZE
}

\section{Abstract}

Agustín Espinosa's critical dimension towards the Canarian literary tradition takes on great importance in his lecture on Viera y Clavijo, "On the sign of Viera", on the occasion of the second centenary of his birth in 1932, in the Circle of Fine Arts of Tenerife. Espinosa's critical gaze of the enlightened archdeacon is analyzed in these pages.

KEYwORDs: Canary literary tradition, 18th century, Agustín Espinosa's literary criticism. 
El siglo XviıI y la literatura canaria fueron objeto del apasionado interés revalorizador del profesor, catedrático de Literatura Española, que fue Agustín Espinosa, tal como se documenta en su tesis doctoral, leída en el Centro de Estudios Históricos en 1924, sobre el impulsor de los espectadores madrileños José Clavijo y Fajardo, y el ensayo objeto de estas páginas, «Sobre el signo de Viera». No puede olvidarse, además, su pionera recolección de romances (Trapero 1987) por el sur de Tenerife, cuando desempeńó una ayudantía en la Universidad de La Laguna, publicados parcialmente en La Rosa de los vientos o en Azor. También se le debe, en su contribución al mejor conocimiento de la literatura y la tradición canarias, su reconocimiento a la función renovadora de Graciliano Afonso en su Oda al Teide.

La tradición insular en el vértice de la retina espinosista está atenta a todo lo que le circunda, tanto del presente como del pasado, sea real, sea imaginado o su yuxtaposición. "Agustín Espinosa, aunque no del todo crítico sustantivo, hace crítica subjetiva recargada de simbolismos; que es todavía la más viviente preocupación por la actualidad literaria de las islas", en palabras de Ramón Feria (1936: 16). Pero también hace crítica objetiva, capaz de extraer las coordenadas culturales, mentales y los fundamentos de la escritura de dos ilustrados isleños como fueron José Clavijo y Fajardo y Viera y Clavijo, dos de las aisladas individualidades de la tradición insular, a juicio también de Ramón Feria. Valbuena Prat, por su parte, en su prólogo a la publicación de Don José Clavijo y Fajardo, al cuidado de Ventura Doreste y Alfonso Armas, nos dice de Espinosa:

Espinosa cantó en prosa poética la isla de sus iniciaciones profesorales, en su precioso y ágil Lancelot $28^{\circ}-7^{\circ}$. Era el tiempo de mis Álvaros y Dominicos, con sus imposibles Venus pirandellianas o los Crucifijos lastimeros de los viejos pasos de Procesión, que Agustín tanto estimara. García Lorca entonaba sus canciones granadinas, y los Ángeles de Alberti se lanzaban al espacio infinito. Gemían en el Albaicín los cantos jondos inéditos de los primeros romances gitanos, y en las islas perdidas florecían las rosas de los vientos. (XI)

Espinosa en clave insular recupera la tradición canaria en el intento denodado de hallar, de construir la propia identidad, que en su tiempo él sustancia en la defunción del mito de Dácil, en palabras propias, en el nacimiento de lo que denominó el «contramito de Dácil». Nos dice que ya no más sinfonías en blanco, de las que no se espera nada, ya no mirar más al mar, sino a la tierra, de la que se espera todo, de la que Espinosa parece esperar también todo, invitándonos a que lo hagamos también nosotros, a que nos sumerjamos en esa corriente revitalizadora, de la que saldrá la nueva identidad canaria.

Asimismo, Espinosa contribuye a fijar ese itinerario histórico desde los viejos romances hasta llegar al siglo XVIII, que nos ocupa ahora, siglo en el que «se casaban los hombres para bailar con sus empelucadas esposas las complicadas danzas de entonces" (1933: 113). Espinosa mira al siglo XVIII, al autor realejero con motivo de la celebración del centenario del nacimiento de Viera y Clavijo, invitado por el Círculo de Bellas Artes para hablar bajo el registro formal de un ensayo de definición, intento a última hora, como afirma. Una hora que se produce en tiempo real, situada 
en su contexto, europeo, hispánico e insular, el de los años treinta del siglo xx, en el que el siglo XVIII va superando solo en parte prejuicios y desprecios tras un Siglo de Oro siempre triunfante, aspecto este determinante en el enjuiciamiento negativo de la centuria ilustrada, del que participan también otros intelectuales coetáneos.

Tras un inicio en el texto en el que se detiene a matizar por qué hay que mirar a Viera no desde abajo, sino desde arriba, sienta la base ideológica que fundamentará su ulterior análisis, la morfología cultural. Espinosa asume la división binaria, clasicismo y barroquismo, que atraviesa toda la historia de la cultura occidental con sus modelos explicitados en el texto, desde Conceptos fundamentales en la Historia del Arte, de Wölffling (1924), Goethe ${ }^{1}$ a Las ideas y las formas. Estudios sobre morfología de la cultura, de Eugenio D'Ors (1928). Comparte plenamente la idea de la cultura occidental y universal, desde Oriente, como la historia de la sucesión de las formas, de los estilos dominantes, clásico y barroco. Cita su teoría de las formas que sean y las formas que vuelan, la manera clásica y la romántica. Su punto de vista sobre la cultura es más biológico que morfológico, nos dice. Pero enfatiza que la cultura ha transitado por oscilaciones pendulares, como bien se aprecia en la naturaleza, a diferencia de D’Ors, que entiende que las formas del espíritu de la sociedad quieren ordenar, instaurar leyes teóricas, introducir la razón allá donde existe lo separado y lo diverso. En ello Espinosa marca un matiz diferenciador haciendo hincapié en "las análogas alternancias a las que rigen el día y la noche, el flujo y el reflujo marinos, la vida y la muerte, la vigilia y el sueńo, el invierno y su opuesto el estío" (1980: 298).

Ha sido, afirma, un continuo regresar, un cambiar de signo matizado por la hora y el momento en que la cronología le ha correspondido. Espinosa asume esta genealogía de la cultura que contempla desde Oriente las dos «voltadas» de péndulo en que por tercera vez se repite el alternado cuento que se produce de nuevo en el siglo XVIII. La cara clásica es el equilibrio, la claridad, la perfección, la cordura, la norma, opuestas «facias» que se corresponden con los conceptos wölfflingianos de lineal, pintoresco, superficial y profundo, cíclico y acíclico, plurilateral y unilateral, estático y dinámico, etcétera. Tras un recorrido por las dos «facias», Espinosa nos invita a detenernos en la hora neoclásica de España.

Se adentra así en su definición de la centuria, vuelta a su primera pasión en su viaje en el tiempo más allá de su tesis doctoral sobre el lancelotiano Clavijo y Fajardo. Un siglo que para un Azorín, que redescubre y reconoce el valor de la centuria, en palabras de Rogelio Reyes Cano:

El siglo XvirI es la puerta de la época moderna, divisoria entre el mundo antiguo y el de nuestros días, fundamento de la mayor parte de los criterios y valores sobre

1 Véase de Agustín Espinosa (1927) su artículo «De Antón Chejov a Eugenio D’Ors», en el que reseña el nuevo libro de D'Ors, Las ideas y las formas, publicado en la Biblioteca de Ensayos de la editorial Páez de Madrid. Afirma que D’Ors esquiva a Goethe como puro soldado de Occidente a favor de Jean Nicod, a propósito de la llegada de una nueva aurora a la cultura occidental. Subraya su ausencia. 
los que se asienta la vida contemporánea, con su criticismo, su inquietud intelectual, su superación del pudor personal y su valoración de los sentimientos, base de la sensibilidad romántica (2001: 119).

¿Ve así Espinosa nuestro siglo XVIII? En parte sí, pero no lo rescata del todo. Su afirmación de que es un fenómeno patológico, que se trata de una centuria enfermiza que no se atreve a vivir de sus propias fuerzas, necesitada de las muletas que le proporciona Francia, políticas, literarias y hasta artísticas y científicas, participa de una visión común a buena parte de la intelectualidad de su tiempo. Aunque su postura sea más optimista que la de Ortega, para quien a España le faltó el siglo XVIII:

Como tercer término en la comparación podemos tomar a Espańa. ¿Qué hallamos? Una escasez de formas dieciochescas [...] Cuanto más se medita sobre nuestra historia, más clara se advierte esta ausencia del siglo xviII. Nos ha faltado el gran siglo educador [...] Este ha sido el triste sino de España, la nación europea que se ha saltado un siglo insustituible (1946: 2297).

Esta visión negativa, en parte asumida también por Espinosa, se debe más al desconocimiento que a otra cosa. Respecto a la poca valoración de la centuria en las primeras décadas del xx, Álvarez Barrientos (2006: 316) explica que la identificación del XVIII como siglo casticista y nacional, con su asiento en la literatura costumbrista o en la tonadilla escénica, en los majos, en los bandoleros, fue el espejo en que se miraron los españoles para crear una identidad basada en la tradición castiza que se opusiera a la extranjerizante modernidad ilustrada, el afrancesamiento, puesto bajo sospecha. Estas dos caras recorren la centuria, tal como se manifestó en las disputas entre los defensores del teatro del Siglo de Oro y los que abogaban por la reforma neoclásica, o como recoge el propio Espinosa en su estudio sobre Clavijo y Fajardo ( Los artículos de Clavijo sobre el teatro y los autos sacramentales hicieron levantar frente al Pensador los ánimos de los nacionalistas», [1970: 58]), al abordar los ataques y las controversias de los que se hace eco su periódico sobre su rechazo a las corridas de toros («Es ésta una de las valientes y calurosas empresas llevadas a cabo por Clavijo, y donde quedan más evidentes su gran talento e ingenio, su vasta ilustración y sus maravillosas condiciones de escritor» [1970: 89]).

Anarquistas, krausistas o socialistas desdeñaron esta lectura negativa de la Ilustración espańola por considerarla propia de los conservadores nacionales, lo que los condujo a exaltar y a leer a Rousseau, a Voltaire, a Diderot, obviando cualquier referencia a los pensadores y creadores españoles del xviıI. De este modo, Ortega se queda con la imagen de un siglo costumbrista, de majos y de toreros, un siglo pobre en literatura, por asumir esta separación, un siglo que no secunda la actuación de Francia, Inglaterra o Alemania, cuando si se mira en detalle la imagen distorsiona el legado del siglo XviII.

La imagen del xviII que defiende Espinosa coincide con la visión de su tiempo, que apunta, por tanto, a la decadencia de los Austrias, producto de un debilitamiento ocasionado por los excesos anteriores. Percepción que se advierte en el propio siglo y a finales del XVII en la visión desengañada de Gracián, de Saavedra 
Fajardo, de los moralistas barrocos, que certificaron los signos y las causas de la crisis barroca, causante según Espinosa de la incapacidad de la centuria siguiente de levantarse por sí sola. Reproduce el diagnóstico de una España enferma que ha de curarse con las muletas y la medicina que viene de Francia. La crítica, como se sabe, hace tiempo que ha estudiado la crisis del siglo XvII, sus razones económicas y políticas. Tras la Guerra de Sucesión, la entrada de una nueva dinastía vino acompañada de un giro, de un cambio de mentalidad. El siglo está recorrido por la voluntad reformista, clave definitoria de buena parte de la prosa desde Feijoo a Jovellanos, que empieza en los últimos años del reinado de Carlos II con el movimiento de los novatores, negando así la parálisis y la decadencia atribuidas inveteradamente. En la revisión de los años finales del siglo XVII ya no se cuestionan, tras las investigaciones de José María López Piñero, las decisivas aportaciones de la ciencia española a finales del Xvir con Juan de Cabriada, entre otros.

La declaración de la absoluta dependencia de Francia que hace Espinosa no se sostiene en la crítica posterior, especialmente con los trabajos señeros a partir de la década de los años cincuenta del siglo xx que dieron un giro a la idea recurrente de un siglo estéril, flojo y prescindible. Historiadores como Domínguez Ortiz, Sánchez Blanco o Pérez Magallón han coincidido en que la influencia alemana e inglesa fue importante en los aspectos estéticos e ideológicos, también la italiana en música y en arquitectura. De hecho, sorprende porque el propio Espinosa atiende en su tesis sobre Clavijo y Fajardo a las fuentes inglesas en El Pensador, Locke o Addison. También menciona la influencia del filósofo en el pensamiento pedagógico de Clavijo junto con la de Rousseau. Aunque sea una cita larga merece la pena leer su comentario a la llegada de Clavijo a la Corte en 1745 :

Llega, pues, Clavijo a la Corte espańola cuando la política de regeneración de los Borbones empezaba a dar sus primeros frutos. Tres años hacía que Fernando VI regía los destinos de Espańa. Recién firmada, casi, la paz de Aquisgrán, el espíritu innovador del Marqués de la Ensenada hacía sentir todo su valer, y el restablecimiento de las antiguas relaciones intelectuales con Europa abría nuevos y amplios horizontes a la intelectualidad española. El Filantropismo invadía las esferas sociales, frente a la tradición imperialista, y con el título de despotismo ilustrado, creaba Academias y realizaba reformas en todos los órdenes. El Clasicismo iniciaba su heroica cruzada contra la agonizante literatura nacionalista y, en fin, todas las corrientes filosóficas y literarias extranjeras empezaban a encontrar numerosos adeptos en España, en cuyo hecho ponía no poca parte el gobierno, apoyando la labor de los reformadores (1970: 20-21).

También hace tiempo que se superó la idea del aislamiento de España, que no estuvo al margen en la recepción del pensamiento europeo, como bien expresa en el párrafo citado. Se fue en busca de Europa para traerla a España, como hace Viera o como hizo Leandro Fernández de Moratín. El espíritu europeizante es parte esencial del proyecto ilustrado con la idea de alcanzar la autonomía de la razón de la que no solo habló Kant, porque también está en Feijoo. Para Espinosa en «Sobre el signo de Viera»: 
El clasicismo del siglo XviII es un clasicismo de segunda mano, por incapacidad de remontarse hasta la fuente original. Es un clasicismo que viene de Europa-Francia, Italia-, de la nueva Europa; es un clasicismo de tono europeizante o europeizador. No se expanden los valores espańoles por Europa como en el siglo Xvir, sino que si el español sale de España es para traerse a Europa al volver. Se produce de fuera adentro y no de dentro afuera. La importación es ahora regente como lo fue antes la exportación [...]. Se hace importador, y justifica así su hora (1980: 300).

Matiza aquí lo que declaró en su tesis sobre Clavijo y Fajardo. El clasicismo renovó la literatura nacional, a la que estigmatiza, pero añade ahora que es de segunda mano. Creo que Espinosa hereda prejuicios sobre la centuria y no reconoce el equilibrio entre tradición, con raíces hispanas, e innovación, con orientación europea, que practicaron casi todos los autores españoles, incluido Viera. Nunca se desatendió la tradición entre los reformadores. Esto es un prejuicio o desconocimiento, como bien afirma Álvarez Barrientos:

El proceso se caracterizó aquí por intentar integrar la tradición en las reformas, o por llevar a cabo estas desde la primera. En este sentido, la Ilustración española tiene bastante de común con la alemana, esencialmente reformista, con censura y sin grandes ataques a la religión (no se quiere irreligión, sino una religión íntima y sincera). Los ilustrados españoles intentaron que España tuviera un papel en Europa, mientras perdía su relevante posición a medida que cedía territorios en sucesivos tratados de paz. Este intento de aunar tradición y progreso se hacía desde la conciencia de encontrarse viviendo en una nueva época que se daba nombre a sí misma, como ya se ha visto, y en la que urgía que el país encontrara un papel europeo, ya que había perdido el que antes representaba de potencia (2005: 19).

Y aquí estribó la dificultad. En ello pesó mucho el valor y la proyección de toda la literatura del Siglo de Oro y la lectura posterior de ser la única portadora de la tradición española, oponiéndose con virulencia a la nueva literatura y a los nuevos aires reformistas.

Comparte Espinosa una imagen del siglo XviıI español que procede en parte de Menéndez Pelayo, con su postura austracista y, por tanto, antiborbónica. Espinosa valora de modo negativo la realidad política que se instaura con los Borbones con una entrada masiva de hábitos, costumbres, modas, autores, ideas, procedentes del exterior. Se construyó una visión de España que buscó en el pasado referencias de grandeza ante la decadencia del presente finisecular del siglo XIX. Los discursos regeneracionistas abundaron en una España átona, que consideró la centuria dominada por el volterianismo y el afrancesamiento, lectura sesgada que se superó a partir de los años cincuenta del siglo xx.

Espinosa, por otra parte, llevó a cabo las primeras tentativas de la crítica insular en clave moderna, con un propósito nuevo frente a los acercamientos de la centuria anterior, como el de crear una tradición que funde las dos «facias» que mencionamos líneas arriba. No obstante, Viera y Clavijo no careció de estudios y reconocimientos críticos durante el siglo XIX. Viera (Galván 2006-2007: 145-149) no dejó de ser un referente en la cultura de las islas e impregnó la cultura escrita de 
la centuria, como en la publicación emblemática que fue la Ilustración canaria, la propuesta editorial de la Imprenta Isleña o las primeras ediciones de su obra.

A continuación en "Sobre el signo de Viera», en el cuarto subapartado, se pregunta Espinosa en qué dirección habría que incluir a Viera, expuesta en líneas precedentes en su análisis, en donde afirma que hay dos aspectos que definen el siglo: el crítico, negativo, demoledor, ejemplificado en la Enciclopedia, y un segundo, el clásico-versallesco. Considera que tiene más encaje en el primero, junto a Feijoo, anunciador de su caudal signo, afirmación que nadie ha puesto en duda hasta el presente (Galván 2007). Su obra periodística, la historia, el diccionario, sus memorias literarias lo refrendan perfectamente.

Menéndez Pelayo es el referente teórico que de nuevo esgrime Espinosa para emitir el juicio de su poesía mediocre, átona, infortunada, extraña a toda poética esencia, vacía de imaginismo y de corazón. Sin embargo, Espinosa, en consonancia con los juicios positivos del filólogo cántabro, nos dice que los mejores primores los ostenta su Historia de Canarias, donde da muestras de su talento para la generación de mitos, tan cara a Espinosa, "cazador de mitos", en palabras de Alfonso Armas (1960: 6). Espinosa declara que la sugerencia procede de Menéndez Pelayo, nada sospechoso de esquividad hacia Viera, quien recomienda el Diccionario de Historia Natural para entender la inteligencia de la botánica isleña en el poema de Viana. Sigue con la cita de Menéndez Pelayo que subrayó las excelencias del Viera historiador, aunque falta a la ironía volteriana al repetir la fábula de Viana de los amores de la infanta Dácil y el capitán Castillo. En este aspecto discrepa Espinosa al proponer una interpretación sugestiva y de largo recorrido cuando dice:

El corazón de Viera florecía en Canarias y su intelecto en el reino de lo Universal. Bien que sonriese el aislado de supersticiones exóticas, mentiras clericales y fabulerías de la ortodoxia oficiante. Pero ¡del mito «dácilo», del perenne signo canario, de la égloga de nuestra Nausicaa.

Si alguna vez el corazón de Viera se rellena de júbilo, es contando el claro cuento de Dácil, o relatando el viaje de Hércules, o la muerte de Guillén Peraza, el príncipe que murió en pecado mortal.

Ante la poesía popular de sus Islas, Viera olvida su prestigio erudito, su severidad de historiógrafo y hace poética historia, y, con el corazón entre sus manos, canta las excelencias de nuestro imberbe folklore.

Canarias lo enciende en la realización poética pura como Europa lo desfoga en cerebrales labores de Universalidad (1980: 301-302).

Espinosa es el primer crítico en interpretar a Viera en esta clave, en apreciar el tono poético, el talento para construir la historia de Canarias como un relato a veces trágico, a veces épico, de penetrar en los límites del mito, especialmente en su obra histórica. Espinosa el mitólogo se muestra receptivo a captar el perfil «insulario", los registros míticos del arcediano. Como dijera Alfonso Armas, son eslabones en la cadena de mitólogos insulares (1960: 52). Las frases finales del ensayo son vibrantes. Sintetizan la admiración y el reconocimiento de la lección de universalidad de Viera desde su signo interior. Conmina a aprender y a llevar a la práctica la lección de que «es necesario que a toda hora subrayemos: Canarias. Frontera 
africana. Atlántica. Ibérica. Universal» (1980: 302). Canarias para decirlo con las palabras de Viera, que Espinosa justifica:

... de manera que solo por un efecto de negligencia geográfica, o por la idea que se suele tener en Europa de todos los países más acá del Estrecho de Gibraltar, han pasado y pasan para con algunos las islas de Canaria por región de la América, y por indianos sus habitantes [...]. Pero ni este dictamen, a que se acostumbró el vulgo, ni la participación de algunos privilegios, comunes a los americanos e isleños, son bastantes títulos para despojar al África del derecho de contar las Canarias entre las posesiones que le cupieron en la partición del orbe de la tierra (1980: 16-17).

Espinosa es también el primer crítico en captar la tricontinentalidad de Viera. Su época histórica determina este acercamiento, por afinidad semántica, por coincidencia en el carácter «insulario» con vocación universal de ambos. Espinosa de este modo se recrea y se ve reflejado en el espejo de la universalidad de Viera.

ReCibido: octubre de 2020; ACEPTADO: noviembre de 2020 


\section{BIBLIOGRAFÍA}

Álvarez Barrientos, J. (2005): Ilustración y Neoclasicismo en las letras españolas, Madrid: Editorial Síntesis.

Álvarez Barrientos, J. (2006): «El siglo xviII, según Menéndez Pelayo», Boletín de la Biblioteca de Menéndez Pelayo, 82, 297-329.

Armas Ayala, A. (1960): Espinosa, cazador de mitos, Puerto de la Cruz: Instituto de Estudios Hispánicos.

Domínguez Ortiz, A. (2016): Carlos III y la España de la Ilustración, Madrid: Alianza Editorial.

D’ors, E. (1922): Las ideas y las formas. Estudios sobre morfología de la cultura, Madrid: Editorial Páez, Biblioteca de Ensayo, n. ${ }^{\circ} 6$.

D’ors, E. (1922): Tres horas en el museo del Prado. Itinerario estético, Madrid: Rafael Caro Raggio.

Espinosa, A. (1927): «De Antón Chejov a Eugenio D’Ors», en José Miguel Pérez Corrales (ed.), Agustin Espinosa. Lancelot, 28 - $^{\circ}$. Textos 1927-1929, Las Palmas: Insoladas, 239-242.

Espinosa, A. (1933): «Diario de un poeta recién casado», en José Miguel Pérez Corrales (ed.), Media hora jugando a los dados. Textos 1932-1933, Las Palmas: Insoladas, 113.

Espinosa, A. (1970): Don José Clavijo y Fajardo, de Agustín Espinosa, Las Palmas: Ediciones del Excmo. Cabildo Insular de Gran Canaria.

Espinosa, A. (1980): «Sobre el signo de Viera», en Alfonso Armas Ayala y José Miguel Pérez Corrales (eds.), Agustín Espinosa. Textos (1927-1936), Santa Cruz de Tenerife: Aula de Cultura de Tenerife.

Feria, R. (1936): «Noticia de la crítica literaria y artística. El ensayo y los estudios históricos», en Signos de Arte y Literatura, capítulo II, Madrid: edición «El Discreto».

Galván GonzÁlez, V. (2006-2007): «La recepción de la Ilustración en Canarias en el siglo XIX», Estudios Canarios: Anuario del Instituto de Estudios Canarios 50-51, 133-156.

Galván González, V. (2007): «Feijoo en la obra de José de Viera y Clavijo», Cuadernos de estudios del siglo XVIII 17, 149-172.

López Piñero, J.M.a (1969): Ciencia y técnica en la sociedad española de los siglos XVI-XVII, Barcelona: Ariel.

López Piñero, J.M. ${ }^{a}$ (1993): "Juan de Cabriada y el movimiento novator de finales del siglo XVII. Reconsideración después de treinta ańos», Asclepio. Revista de Historia de la Medicina y de la ciencia XLV-1, 3-53.

Ortega y Gasset, J. (1946): «El siglo xvini, educador», en Obras completas, tomo II, 1916-1934, Madrid: Revista de Occidente, 2.927-2.931.

Pérez Magallón, J. (2002): Construyendo la modernidad, la cultura española en el «tiempo de los novatores» (1675-1725), Madrid: Consejo Superior de Investigaciones Científicas.

Reyes Cano, R. (2001): "Azorín o la revalorización del siglo XviII», en De Blanco White a la Generación del 27. Estudios de Literatura Española Contemporánea, Sevilla: Universidad de Sevi1la, 115-129.

SÁnchez-Blanco, F. (1991): Europa y el pensamiento español del siglo XVIII, Madrid: Alianza Editorial.

Trapero, M. (1987-1988): «Agustín Espinosa, primer investigador del romancero canario», Revista de Filología de la Universidad de La Laguna 6-7: 431-455. 
Valbuena Prat, Á. (1970): «Prólogo», en Don José Clavijo y Fajardo, de Agustín Espinosa, Las Palmas de Gran Canaria: Ediciones del Excmo. Cabildo Insular de Gran Canaria.

Wölfflin, H. (1924): Conceptos fundamentales en la Historia del Arte, Madrid: Espasa-Calpe. 